\title{
Citas y apuntes corológicos de interés en ciperáceas ibéricas
}

\section{Jiménez-Mejías, P. ${ }^{1,2}$, Calleja, J.A. ${ }^{1,2}$, Martín-Torrijos, L. ${ }^{3}$, Otero, A. ${ }^{4}$ Martín-Bravo, S. ${ }^{5}$}

1Dpto. de Biología (Botánica), Universidad Autónoma de Madrid, Campus Cantoblanco, Madrid 28049, (España)

${ }^{2}$ Centro de Investigación en Biodiversidad y Cambio Global (CIBC-UAM), Universidad Autónoma de Madrid, Madrid 28049, (España)

${ }^{3}$ Dpto. de Micología, Real Jardín Botánico-CSIC (RJB-CSIC), Plaza Murillo 2, Madrid, (España)

${ }^{4}$ Dpto. de Biodiversidad y Conservación, Real Jardín Botánico-CSIC (RJB-CSIC), Plaza Murillo 2, Madrid, (España)

${ }^{5}$ Área de Botánica, Dpto. de Biología Molecular e Ingeniería Bioquímica, Universidad Pablo de Olavide, ctra. de Utrera km1, 41013, Sevilla (España)

\section{Correspondencia}

P. Jiménez Mejías

e-mail: pjimmej@gmail.com pedro.jimenez@uam.es

Recibido: 28 septiembre 2020

Aceptado: 23 octubre 2020

Publicado on-line: 12 noviembre 2020

Editado por: Marta Recio Criado

\begin{abstract}
Resumen
Se presentan novedades y correcciones corológicas para cuatro especies de ciperáceas ibéricas de interés.
\end{abstract}

Palabras clave: Asturias, Carex, Cordillera Cantábrica, Madrid, Palencia, Scirpus, Sistema Ibérico.

\section{Abstract}

Notes and chorological citations of interest in Iberian ciperaceae.

We present chorological additions and corrections for four species of Iberian sedges of interest.

Keywords: Asturias, Carex, Cantabrian Range, Madrid, Palencia, Scirpus, Iberian System Range

Presentamos nuevos registros de interés para cuatro especies de ciperáceas ibéricas. Aclaramos la distribución de Carex elata All. y C. reuteriana Boiss. en el norte de la Península, donde citas previas de la provincia de Palencia parecen ser errores de identificación. Los materiales testigo se han depositado en el herbario de la Universidad Pablo de Olavide (UPOS).

Carex depauperata Curtis ex Woodw. ESPAÑA, ASTURIAS. Entrago, Senda del Oso dirección norte, río Teverga, bosque de ribera, 43,185599 N 6,079678 W, ca. 400 m, 3 Ago 2020, P. Jiménez-Mejías, L. Martín-Torrijos y A. Otero Gómez (77PJM20, UPOS-13956).

Especie euroasiática que aparece de forma poco abundante, muy dispersa y localizada por toda la mitad norte de la Península lbérica (Luceño, 2008). Ha sido citada recientemente de una única localidad en Portugal (Jansen \& Koopman, 2014). Primera cita para Asturias y toda la vertiente norte de la Cordillera Cantábrica. Otros hallazgos recientes relevantes para la corología ibérica de esta especie han sido las citas para las provincias de Guadalajara (Mateo Sanz y Pisco García, 1997), León (Molina et al., 2006), Madrid (Baonza Díaz, 2012) y Teruel (Mateo Sanz et al., 2005).

\section{"Carex elata All."}

Corregimos la distribución de C. elata en la provincia de Palencia. Citada del piedemonte de la Cordillera Cantábrica de una localidad asociada a la cuenca alta del río Carrión por Jiménez-Mejías et al.
(2007), dicha cita se basó, desafortunadamente, en un material incompleto que ahora, tras muestreos recientes (2020), podemos confirmar sin ninguna duda como C. nigra (L.) Reichard. Aunque se trata de ejemplares anormalmente grandes (con medidas en torno a los máximos dados para la especie por Luceño \& Jiménez-Mejias, 2008), la combinación de los utrículos papilosos con hojas anfistomáticas descartan por completo a $C$. elata o $C$. reuteriana Boiss. y delata que se trata de poblaciones de $C$. nigra. Otros caracteres anormales de estas plantas, como son las largas vainas basales pardo oscuro (en lugar de cortas y de color pardo rojizo) y el hecho de que formen macollas más o menos definidas, podrían evidenciar cierta introgresión por parte de C. acuta L., que aparece en la cuenca alta del Pisuerga (ver el epígrafe de $C$. reuteriana más abajo), lo que quedaría pendiente de confirmación mediante datos moleculares.

Las localidades confirmadas más cercanas de $C$. elata respecto al norte palentino se encuentran en las estribaciones orientales de la Cordillera Cantábrica, en el río Cadagua y afluentes (Burgos/Vizcaya; Aseginolaza, et al. 1985; Bariego \& Gastón, 2002) o en los páramos burgaleses (Galán, 1990) y ya en plena depresión del río Duero, en Cordovilla la Real (Palencia; Jiménez-Mejías et al., 2007).

Materiales estudiados pertenecientes a Carex nigra. ESPAÑA, PALENCIA. Villalba de Guardo, Laguna de En medio, $1150 \mathrm{~m}, 42^{\circ} 44^{\prime} \mathrm{N} 4^{\circ} 47^{\prime} \mathrm{W}, 25 \mathrm{Jul} 2001, F$. Cabezas \& C. Aedo (LEB-091503, MA 695429); Ibidem, prados temporalmente inundados en los 
márgenes de la laguna, $42.735732-4.789038,1150$ m, 28 Ago 2020, S. Martín-Bravo (62SMB20, UPOS13957).

\section{Carex montana L.}

ESPAÑA, SORIA. Sierra Cebollera, Puerto de Piqueras, pinar laxo de Pinus sylvestris con sotobosque de brezo, $42.052646 \mathrm{~N} 2.540489 \mathrm{~W}$, 1580 m, 17 Jul 2020, P. Jiménez-Mejías y J.A. Calleja (46PJM20, UPOS-13958).

Especie euroasiática conocida de los pisos montano y subalpino del eje pirenaico-cantábrico (Luceño, 2008). Primera cita para Soria y el Sistema Ibérico. Probablemente su distribución sea más amplia en el complejo de las sierras de Cebollera, Urbión, Cameros y Neila penetrando así en La Rioja.

Carex pendula Huds.

ESPANA, MADRID. Senda Real entre Madrid y Aranjuez, poco antes de la M-40, a la altura de la Caja Mágica, río Manzanares, orillas del río, en olmeda riparia de Ulmus cf. minor, aparentemente espontánea, 4022.1953`N 340.9258’W, 490 m, 16 Ago 2020, S. Martín-Bravo \& A.M. Rodríguez Saéz (24SMB20, UPOS-13959).

Especie de cárice de gran porte ampliamente distribuida por Europa occidental (Míguez et al., 2017, 2018), a menudo utilizada como ornamental. Relativamente frecuente en la península, aparece sobre todo en hábitats termófilos y húmedos, especialmente en bosques riparios, siendo mucho más rara hacia la zona centro (Luceño, 2008; Sánchez-Villegas et al., 2019), de donde existen citas recientes. Ésta supone la primera cita para la provincia de Madrid, donde su presencia parece ser aparentemente nativa y consta de un único individuo observado.

\section{"Carex reuteriana Boiss."}

Corregimos la distribución de $C$. reuteriana, endemismo ibero-magrebí, en el norte de la Península Ibérica, donde es la subespecie tipo la que está presente. La otra subespecie reconocida para el taxon, la subsp. mauritanica (Boiss. \& Reut.) Jiménez-Mejías \& Luceño, se distribuye por el suroeste de la Península lbérica y puntos aislados del noroeste de África (Benítez-Benítez et al., 2018). La presencia de Carex reuteriana subsp. reuteriana ha sido descrita en la vertiente sur del extremo occidental y del centro de la Cordillera Cantábrica, en las provincias de León y Palencia (Luceño \& JiménezMejías, 2008). Sin embargo, nuestras prospecciones recientes revelan que las localidades citadas en la cuenca alta del Pisuerga (Herrero Cembranos, 1989) corresponden sin ninguna duda a $C$. acuta.

Otras citas referenciadas por los autores del tratamiento de Flora Iberica (www.anthos.es) pero de la cuenca alta del Carrión han resultado corresponder a materiales de $C$. nigra. Excluimos por tanto $C$. reuteriana subsp. reuteriana de la provincia de Palencia. De este modo el límite oriental de esta especie en la Cordillera Cantábrica quedaría fijado en la provincia de León.

Materiales estudiados pertenecientes a Carex acuta. ESPAÑA, PALENCIA. Cervera de Pisuerga, Ligüerzana, río Pisuerga, aguas abajo de Cervera, orillas despejadas, $42.853759 \mathrm{~N} 4.445655 \mathrm{~W}, 970 \mathrm{~m}, 18 \mathrm{Jul}$ 2020, P. Jiménez-Mejías y J.A. Calleja (68PJM20, UPOS-13960). Cervera de Pisuerga, Celada de Roblecedo, $1 \mathrm{~km}$ antes del pueblo, río Castillería, bordes del río, 42 ${ }^{\circ} 56^{\prime} 47.1^{\prime \prime} \mathrm{N} 4^{\circ} 26^{\prime} 29.5^{\prime \prime} \mathrm{W}, 1160 \mathrm{~m}$, 28 Ago 2020, S. Martín-Bravo (57SMB20, UPOS13961). San Salvador de Cantamuda, Venta Morena, río Pisuerga, orillas del río, 4256'50.56"N 4²9'29.74"W, 1085 m, 28 Ago 2020, S. Martín-Bravo (58SMB20, UPOS-13962). San Salvador de Cantamuda, entre el desvío a Los Llazos y Tremaya, río Pisuerga, orillas del río, 42 $58^{\circ} 48.3^{\prime \prime} \mathrm{N} 4^{\circ} 28^{\prime} 10.9^{\prime \prime} \mathrm{W}$, 1150 m, 28 Ago 2020, S. Martín-Bravo (60SMB20, UPOS-13963).

Materiales estudiados pertenecientes a Carex nigra.

Villanueva de Abajo, arroyo de Valdemorata, [30TUN63], $1060 \mathrm{~m}, 26-\mathrm{VII}-2001$, F. Cabezas \& C. Aedo (MA-695381).

\section{Scirpus sylvaticus $L$.}

ESPAÑA, PALENCIA. Quintanaluengos, río Pisuerga, orillas fangosas bajo el puente de la PP-2128, $42.856171 \mathrm{~N} 4.423173 \mathrm{~W}, 960 \mathrm{~m}, 18 \mathrm{Jul} 2020, P$. Jiménez-Mejías y J.A. Calleja (66PJM20, UPOS13964).

Especie euroasiática, muy rara, dispersa y localizada en el norte de la Península Ibérica (MartínBravo y Luceño, 2008). Segunda cita para la provincia de Palencia, de donde se había reportado del exclave oriental de Berzosilla unos $40 \mathrm{~km}$ más al este (Rodríguez-García et al. 2015). La cita tiene relevancia desde el punto de vista de la conservación regional de esta especie, ya que está recogida en el Decreto 63/2007, por el que se crea el Catálogo de Flora Protegida de Castilla y León, en la categoría de "especies de atención preferente".

\section{Agradecimientos}

Agradecemos a C. Barciela (herbario UPOS) por el apoyo técnico, a A.M. Rodríguez Sáez la ayuda prestada durante el trabajo de campo y a M. Luceño y un revisor anónimo por sus comentarios sobre una versión previa de la presente nota.

\section{Bibliografía}

Aseginolaza C., Gomez, D., Lizaur, X., Montserrat, G., Morante, G., Salaverria, M.R., UribeEchebarria P.M. \& J.A. Alejandre, J.A. (1985). Araba, Bizkaia eta Gipuzkoako landare katalogoa. Catálogo florístico de Álava, Vizcaya y Guipúzcoa, Gobierno Vasco. Departamento de Política Territorial y Transportes. Vitoria-Gasteiz. 
Baonza, J. (2012). Citas de flora vascular de interés de la Sierra del Rincón (Madrid, España). Botanica Complutensis, 36, 105-111.

Bariego, P. \& Gastón, A. (2002). Catálogo florístico de los Montes de Ordunte (Burgos, España). Ecología, 16, 97-152. http://dx.doi.org/10.5209/

Benítez-Benítez, C., Escudero, M., RodríguezSánchez, F., Martín-Bravo, S. \& Jiménez-Mejías, P. (2018). Pliocene-Pleistocene ecological niche evolution shapes the phylogeography of a Mediterranean plant group. Molecular Ecology, 27, 1696-1713. https://doi.org/10.1111/mec. 14567

Galán, P. (1990). Contribución al estudio florístico de las comarcas de la Lora y Páramo de Masa (Burgos). Fontqueria, 30, 1-167.

Herrero Cembranos, L. (1989). Flora y vegetación de la margen izquierda de la cuenca alta del río Pisuerga (Palencia), Tesis doctoral. Universidad de León.

Jansen, J. \& Koopman, J. (2014). Carex depauperata Curtis ex Stokes (Cyperaceae), a new species for Portugal. Silva Lusitana, 22(1), 135-137.

Jiménez-Mejías, P., Escudero, M., Chaparro, A.J. \& Luceño, M. (2007). Notulae, taxinomicae, chorologicae, nomenclaturales, bibliographicae aut philologicae in opus "Flora lberica" intendentes (12-14). 12. Novedades corológicas del género Carex para la Península Ibérica, Acta Botanica Malacitana, 32, 305-309.

Luceño, M. (2008). Carex. In S. Castroviejo, M. Luceño, A. Galán de Mera, P. Jiménez Mejías, F.J. Cabezas \& L. Medina (Eds.), Flora Iberica 18 (pp. 109-250). Real Jardín Botánico, CSIC. Madrid.

Luceño, M. \& Jiménez-Mejías, P. (2008). Carex sect. Phacocystis. In S. Castroviejo, M. Luceño, A. Galán de Mera, P. Jiménez Mejías, F.J. Cabezas \& L. Medina (Eds.), Flora Iberica 18 (pp. 237-246). Real Jardín Botánico, CSIC. Madrid.

Martín-Bravo, S. \& Luceño, M. (2008). Scirpus. In S. Castroviejo, M. Luceño, A. Galán de Mera, P. Jiménez Mejías, F.J. Cabezas \& L. Medina (Eds.),
Flora Iberica 18 (pp. 34-36). Real Jardín Botánico, CSIC. Madrid.

Mateo Sanz, G. \& Pisco García, J.M. (1997). Contribuciones a la flora del sistema ibérico, XII. Flora Montiberica, 5, 47-49.

Mateo Sanz, G., Fabado, J. \& Torres, C. (2005). Novedades florísticas procedentes del término de Noguera de Albarracín (Teruel). Flora Montiberica, 30, 63-68.

Míguez, M., Gehrke, B., Maguilla, E., Jiménez-Mejías, P. \& Martín-Bravo, S. (2017). Carex sect. Rhynchocystis (Cyperaceae): a Miocene subtropical relict in the Western Palaearctic showing a dispersal-derived Rand Flora pattern. Journal of Biogeography, 44, 2211-2224

https://doi.org/10.1111/jbi.13027

Míguez, M., Martín-Bravo, S. \& Jiménez-Mejías, P. (2018). Reconciling morphology and phylogeny allows an integrative taxonomic revision of the giant sedges of Carex section Rhynchocystis (Cyperaceae). Botanical Journal of the Linnean Society, 188, 34-58. https://doi.org/10.1093/botlinnean/boy040

Molina, A.M., Acedo, C. \& Llamas, F. (2006). Observaciones sobre el género Carex en la provincia de León (NW España). Lagascalia, 26, 25-37.

Rodríguez-García, A., Alfaro-Saiz, E., AlonsoRedondo, R. \& García-González, M.E. (2015). Aportaciones a la flora de las zonas húmedas de la provincia de Palencia, II. Flora Montiberica, 61, 124-130.

Sánchez-Villegas, R., Sánchez-Villegas, M., Robles, J.L., Benz, C., Sánchez, L., Martínez, B. Hernández, R., Valducei, M.A., Márquez, J.I, Rico, J.C., Estévez, F., Sánchez-Amador, E., De Sande, F.J., Marín, P., Rico, E. \& Luceño, M. (2019). Novedades corológicas para la flora vascular de la sierra de Gredos (Sistema Central, España). Flora Montiberica, 75, 101-110. 